\title{
ON HOPF GALOIS HIRATA EXTENSIONS
}

\author{
GEORGE SZETO and LIANYONG XUE
}

Received 17 March 2003

\begin{abstract}
Let $H$ be a finite-dimensional Hopf algebra over a field $k, H^{*}$ the dual Hopf algebra of $H$, and $B$ a right $H^{*}$-Galois and Hirata separable extension of $B^{H}$. Then $B$ is characterized in terms of the commutator subring $V_{B}\left(B^{H}\right)$ of $B^{H}$ in $B$ and the smash product $V_{B}\left(B^{H}\right) \# H$. A sufficient condition is also given for $B$ to be an $H^{*}$ Galois Azumaya extension of $B^{H}$.
\end{abstract}

2000 Mathematics Subject Classification: 16W30, 16H05.

1. Introduction. Let $H$ be a finite-dimensional Hopf algebra over a field $k$, $H^{*}$ the dual Hopf algebra of $H$, and $B$ a right $H^{*}$-Galois extension of $B^{H}$. In [3], the class of $H^{*}$-Galois Azumaya extensions was investigated and in [8], it was shown that $B$ is a Hirata separable extension of $B^{H}$ if and only if the commutator subring $V_{B}\left(B^{H}\right)$ of $B^{H}$ in $B$ is a left $H$-Galois extension of $C$, where $C$ is the center of $B$ (see [8, Lemma 2.1, Theorem 2.6]). The purpose of the present paper is to characterize a right $H^{*}$-Galois and Hirata separable extension $B$ of $B^{H}$ in terms of the commutator subring $V_{B}\left(B^{H}\right)$ and the smash product $V_{B}\left(B^{H}\right) \# H$. Let $B$ be a right $H^{*}$-Galois extension of $B^{H}$ such that $B^{H}=B^{H^{*}}$. Then the following statements are equivalent:

(1) $B$ is a Hirata separable extension of $B^{H}$,

(2) $V_{B}\left(B^{H}\right)$ is an Azumaya $C$-algebra and $V_{B}\left(V_{B}\left(B^{H}\right)\right)=B^{H}$,

(3) $V_{B}\left(B^{H}\right)$ is a right $H^{*}$-Galois extension of $C$ and a direct summand of $V_{B}\left(B^{H}\right) \# H$ as a $V_{B}\left(B^{H}\right)$-bimodule,

(4) $V_{B}\left(B^{H}\right)$ is a right $H^{*}$-Galois extension of $C$ and $V_{B}\left(B^{H}\right) \# H$ is a direct summand of a finite direct sum of $V_{B}\left(B^{H}\right)$ as a bimodule over $V_{B}\left(B^{H}\right)$. Moreover, an equivalent condition is given for a right $H^{*}$-Galois and Hirata separable extension $B$ of $B^{H}$ to be an $H^{*}$-Galois Azumaya extension which was studied in [3, 7]. Also, let $B$ be a right $H^{*}$-Galois and Hirata separable extension of $B^{H}$ and $A$ a subalgebra of $B^{H}$ over $C$ such that $B^{H}$ is a projective Hirata separable extension of $A$ containing $A$ as a direct summand as an $A$-bimodule. Then $V_{B^{H}}(A)$ is a separable subalgebra of $B^{H}$ over $C$, and there exists an $H^{-}$ submodule algebra $D$ in $B$ which is separable over $C$ such that $D^{H}=V_{B^{H}}(A)$ and $D \cong V_{B^{H}}(A) \otimes_{Z} F$ as Azumaya $Z$-algebras, where $Z$ is the center of $D$ and $F$ is an Azumaya $Z$-algebra in $D$. 
2. Basic definitions and notations. Throughout, $H$ denotes a finite-dimensional Hopf algebra over a field $k$ with comultiplication $\Delta$ and counit $\varepsilon, H^{*}$ the dual Hopf algebra of $H, B$ a left $H$-module algebra, $C$ the center of $B, B^{H}=\{b \in$ $B \mid h b=\varepsilon(h) b$ for all $h \in H\}$ which is called the $H$-invariants of $B$, and $B \# H$ the smash product of $B$ with $H$, where $B \# H=B \otimes_{k} H$ such that for all $b \# h$ and $b^{\prime} \# h^{\prime}$ in $B \# H,(b \# h)\left(b^{\prime} \# h^{\prime}\right)=\sum b\left(h_{1} b^{\prime}\right) \# h_{2} h^{\prime}$, where $\Delta(h)=\sum h_{1} \otimes h_{2}$. The ring $B$ is called a right $H^{*}$-Galois extension of $B^{H}$ if $B$ is a right $H^{*}$-comodule algebra with structure map $\rho: B \rightarrow B \otimes_{k} H^{*}$ such that $\beta: B \otimes_{B^{H}} B \rightarrow B \otimes_{k} H^{*}$ is a bijection, where $\beta(a \otimes b)=(a \otimes 1) \rho(b)$.

For a subring $A$ of $B$ with the same identity 1, we denote the commutator subring of $A$ in $B$ by $V_{B}(A)$. We call $B$ a separable extension of $A$ if there exist $\left\{a_{i}, b_{i}\right.$ in $B, i=1,2, \ldots, m$, for some integer $\left.m\right\}$ such that $\sum a_{i} b_{i}=1$ and $\sum b a_{i} \otimes b_{i}=\sum a_{i} \otimes b_{i} b$ for all $b$ in $B$, where $\otimes$ is over $A$. An Azumaya algebra is a separable extension of its center. A ring $B$ is called a Hirata separable extension of $A$ if $B \otimes_{A} B$ is isomorphic to a direct summand of a finite direct sum of $B$ as a $B$-bimodule. A right $H^{*}$-Galois extension $B$ is called an $H^{*}$-Galois Azumaya extension if $B$ is separable over $B^{H}$ which is an Azumaya algebra over $C^{H}$. A right $H^{*}$-Galois extension $B$ of $B^{H}$ is called an $H^{*}$-Galois Hirata extension if $B$ is also a Hirata separable extension of $B^{H}$. Throughout, an $H^{*}$-Galois extension means a right $H^{*}$-Galois extension unless it is stated otherwise.

3. The $H^{*}$-Galois Hirata extensions. In this section, we will characterize an $H^{*}$-Galois Hirata extension $B$ of $B^{H}$ in terms of the commutator subring $V_{B}\left(B^{H}\right)$ of $B^{H}$ in $B$ and the smash product $V_{B}\left(B^{H}\right) \# H$. A relationship between an $H^{*}$-Galois Hirata extension and an $H^{*}$-Galois Azumaya extension is also given. We begin with some properties of an $H^{*}$-Galois Hirata extension $B$ of $B^{H}$. Throughout, we assume $B^{H}=B^{H^{*}}$.

LEMMA 3.1. If $A_{1}$ and $A_{2}$ are $H^{*}$-Galois extensions such that $A_{1}^{H}=A_{2}^{H}$ and $A_{1} \subset A_{2}$, then $A_{1}=A_{2}$.

Proof. By [3, Theorem 5.1], there exist $\left\{x_{i}, y_{i} \in A_{1} \mid i=1,2, \ldots, n\right\}$ for some integer $n$ such that, for all $h \in H, \sum x_{i}\left(h y_{i}\right)=T(h) 1_{A_{1}}$, where $T \in \int_{H^{*}}^{r}$, the set of right integrals in $H^{*}$. Let $t \in \int_{H}^{l}$, the set of left integrals in $H$, such that $T(t)=1$, then $\left\{x_{i}, f_{i}=t\left(y_{i}-\right) \mid i=1,2, \ldots, n\right\}$ is a dual basis of the finitely generated and projective right module $A_{1}$ over $A_{1}^{H}$. Since $A_{1} \subset A_{2}$ such that $A_{1}^{H}=A_{2}^{H},\left\{x_{i}, f_{i} \mid i=1,2, \ldots, n\right\}$ is also a dual basis of the finitely generated and projective right module $A_{2}$ over $A_{1}^{H}$. This implies that $A_{1}=A_{2}$.

LEMMA 3.2. If $B$ is an $H^{*}$-Galois Hirata extension of $B^{H}$, then $B^{H}$ is a direct summand of $B$ as a $B^{H}$-bimodule.

Proof. We use the argument as given in [2]. Since $B$ is an $H^{*}$-Galois and a Hirata separable extension of $B^{H}, V_{B}\left(B^{H}\right)$ is a left $H$-Galois extension of $C$ (see [8, Lemma 2.1, Theorem 2.6]). Hence, $V_{B}\left(B^{H}\right)$ is a finitely generated and 
projective module over $C$ (see [3, Theorem 2.2]). Let $\Omega=\operatorname{Hom}_{C}\left(V_{B}\left(B^{H}\right), V_{B}\left(B^{H}\right)\right)$. Since $C$ is commutative, $V_{B}\left(B^{H}\right)$ is a progenerator of $C$. Thus, $B$ is a right $\Omega$-module such that $B \cong V_{B}\left(B^{H}\right) \otimes_{C} \operatorname{Hom}_{\Omega}\left(V_{B}\left(B^{H}\right), B\right) \cong V_{B}\left(B^{H}\right) \otimes_{C} B^{H^{*}}$ as $C$ algebras, where $f(1) \in B^{H^{*}}$ for each $f \in \operatorname{Hom}_{\Omega}\left(V_{B}\left(B^{H}\right), B\right)$ by the proof of [2, Lemma 2.8]. But $V_{B}\left(V_{B}\left(B^{H}\right)\right)=B^{H}$ (see [2, Lemma 2.5]), so $B \cong V_{B}\left(B^{H}\right) \otimes_{C} B^{H}$. This implies that $V_{B}\left(B^{H}\right)$ is an $H^{*}$-Galois extension of $C$ (see [2, Lemma 2.8]); and so $C$ is a direct summand of $V_{B}\left(B^{H}\right)$ as a $C$-bimodule (see [2, Corollaries 1.9 and 1.10]). Therefore, $B^{H}$ is a direct summand of $B$ as a $B^{H}$-bimodule.

By the proof of Lemma 3.2, $V_{B}\left(B^{H}\right)$ is an $H^{*}$-Galois extension of $C$.

COROLlary 3.3. If $B$ is an $H^{*}$-Galois Hirata extension of $B^{H}$, then $V_{B}\left(B^{H}\right)$ is an $H^{*}$-Galois extension of $C$.

COROLlary 3.4. If $B$ is an $H^{*}$-Galois Hirata extension of $B^{H}$, then $B=B^{H}$. $V_{B}\left(B^{H}\right)$ and the centers of $B, B^{H}$, and $V_{B}\left(B^{H}\right)$ are the same $C$.

Proof. By Corollary 3.3, $V_{B}\left(B^{H}\right)$ is an $H^{*}$-Galois extension of $C$, so $B^{H}$. $V_{B}\left(B^{H}\right)$ is also an $H^{*}$-Galois extension of $B^{H}\left(=\left(B^{H} \cdot V_{B}\left(B^{H}\right)\right)^{H}\right)$ with the same Galois system as $V_{B}\left(B^{H}\right)$ (see [3, Theorem 5.1]). Noting that $B^{H} \cdot V_{B}\left(B^{H}\right) \subset B$, we conclude that $B=B^{H} \cdot V_{B}\left(B^{H}\right)$ by Lemma 3.1. Moreover, $V_{B}\left(V_{B}\left(B^{H}\right)\right)=B^{H}$ (see [8, Lemma 2.5]), so the centers of $B^{H}, V_{B}\left(B^{H}\right)$, and $B$ are the same $C$.

THEOREM 3.5. Let $B$ be an $H^{*}$-Galois extension of $B^{H}$. The following statements are equivalent:

(1) $B$ is a Hirata separable extension of $B^{H}$,

(2) $V_{B}\left(B^{H}\right)$ is an $H^{*}$-Galois extension of $C$ and a direct summand of $V_{B}\left(B^{H}\right) \# H$ as a $V_{B}\left(B^{H}\right)$-bimodule,

(3) $V_{B}\left(B^{H}\right)$ is an Azumaya $C$-algebra and $V_{B}\left(V_{B}\left(B^{H}\right)\right)=B^{H}$,

(4) $V_{B}\left(B^{H}\right)$ is an $H^{*}$-Galois extension of $C$ and $V_{B}\left(B^{H}\right) \# H$ is a direct summand of a finite direct sum of $V_{B}\left(B^{H}\right)$ as a bimodule over $V_{B}\left(B^{H}\right)$.

Proof. $(1) \Rightarrow(3)$. Since $B$ is an $H^{*}$-Galois and a Hirata separable extension of $B^{H}$, by Lemma 3.2, $B^{H}$ is a direct summand of $B$ as a $B^{H}$-bimodule. Thus, $V_{B}\left(V_{B}\left(B^{H}\right)\right)=B^{H}$ and $V_{B}\left(B^{H}\right)$ is a separable $C$-algebra (see [4, Propositions 1.3 and 1.4]). But the center of $V_{B}\left(B^{H}\right)$ is $C$ by Corollary 3.4 , so $V_{B}\left(B^{H}\right)$ is an Azumaya $C$-algebra.

$(3) \Rightarrow(1)$. Since $V_{B}\left(B^{H}\right)$ is an Azumaya $C$-algebra and $B$ is a bimodule over $V_{B}\left(B^{H}\right), B \cong V_{B}\left(B^{H}\right) \otimes_{C} V_{B}\left(V_{B}\left(B^{H}\right)\right)=V_{B}\left(B^{H}\right) \otimes_{C} B^{H}$ as a bimodule over $V_{B}\left(B^{H}\right)$ (see [1, Corollary 3.6, page 54]). Noting that $B \cong V_{B}\left(B^{H}\right) \otimes_{C} B^{H}$ is also an isomorphism as $C$-algebras and that $V_{B}\left(B^{H}\right)$ is an Azumaya $C$-algebra, we conclude that $V_{B}\left(B^{H}\right) \otimes_{C} B^{H}$ is a Hirata separable extension of $B^{H}$; and so $B$ is a Hirata separable extension of $B^{H}$.

$(3) \Rightarrow(2)$. By the proof of $(3) \Rightarrow(1), B \cong V_{B}\left(B^{H}\right) \otimes_{C} B^{H}$ such that $V_{B}\left(B^{H}\right)$ is a finitely generated and projective module over $C$, so $V_{B}\left(B^{H}\right)$ is an $H^{*}$-Galois extension of $C$ (see [2, Lemma 2.8]). Moreover, since $V_{B}\left(B^{H}\right)$ is an Azumaya 
$C$-algebra, $V_{B}\left(B^{H}\right)$ is a direct summand of $V_{B}\left(B^{H}\right) \otimes_{C}\left(V_{B}\left(B^{H}\right)\right)^{\circ}$ as a $V_{B}\left(B^{H}\right)$ bimodule, where $\left(V_{B}\left(B^{H}\right)\right)^{\circ}$ is the opposite algebra of $V_{B}\left(B^{H}\right)$. But $V_{B}\left(B^{H}\right) \otimes_{C}$ $\left(V_{B}\left(B^{H}\right)\right)^{\circ} \cong \operatorname{Hom}_{C}\left(V_{B}\left(B^{H}\right), V_{B}\left(B^{H}\right)\right) \cong V_{B}\left(B^{H}\right) \# H$ (see [3, Theorem 2.2]), so $V_{B}\left(B^{H}\right)$ is a direct summand of $V_{B}\left(B^{H}\right) \# H$ as a $V_{B}\left(B^{H}\right)$-bimodule.

$(2) \Rightarrow(3)$. Since $V_{B}\left(B^{H}\right)$ is an $H^{*}$-Galois extension of $C, B^{H} \cdot V_{B}\left(B^{H}\right)$ is an $H^{*}$ Galois extension of $\left(B^{H} \cdot V_{B}\left(B^{H}\right)\right)^{H}$. But $\left(B^{H} \cdot V_{B}\left(B^{H}\right)\right)^{H}=B^{H}$, so $B^{H} \cdot V_{B}\left(B^{H}\right)$ and $B$ are $H^{*}$-Galois extensions of $B^{H}$ such that $B^{H} \cdot V_{B}\left(B^{H}\right) \subset B$. Hence, $B^{H}$. $V_{B}\left(B^{H}\right)=B$ by Lemma 3.1. Thus, the centers of $B$ and $V_{B}\left(B^{H}\right)$ are the same $C$. Moreover, $V_{B}\left(B^{H}\right)$ is a direct summand of $V_{B}\left(B^{H}\right) \# H$ as a $V_{B}\left(B^{H}\right)$-bimodule by hypothesis, so it is a separable $C$-algebra (see [3, Theorem 2.3]). Thus, $V_{B}\left(B^{H}\right)$ is an Azumaya $C$-algebra. But then $B \cong V_{B}\left(B^{H}\right) \otimes_{C} V_{B}\left(V_{B}\left(B^{H}\right)\right)$. On the other hand, by hypothesis, $V_{B}\left(B^{H}\right)$ is an $H^{*}$-Galois extension of $C$, so $B \cong V_{B}\left(B^{H}\right) \otimes_{C} B^{H}$ (see [2, Lemma 2.8]). Therefore, $V_{B}\left(V_{B}\left(B^{H}\right)\right)=B^{H}$.

$(3) \Leftrightarrow(4)$. Since $V_{B}\left(B^{H}\right)$ is an $H^{*}$-Galois extension of $C$, it is a finitely generated and projective module over $C$ and $\operatorname{Hom}_{C}\left(V_{B}\left(B^{H}\right), V_{B}\left(B^{H}\right)\right) \cong V_{B}\left(B^{H}\right) \# H$ (see [3, Theorem 2.2]). But then $V_{B}\left(B^{H}\right)$ is a Hirata separable extension of $C$ if and only if $V_{B}\left(B^{H}\right) \# H$ is a direct summand of a finite direct sum of $V_{B}\left(B^{H}\right)$ as a bimodule over $V_{B}\left(B^{H}\right)$ (see [5, Corollary 3]). Thus, $V_{B}\left(B^{H}\right)$ is an Azumaya $C$-algebra if and only if $V_{B}\left(B^{H}\right)$ is an $H^{*}$-Galois extension of $C$ and $V_{B}\left(B^{H}\right) \# H$ is a direct summand of a finite direct sum of $V_{B}\left(B^{H}\right)$ as a bimodule over $V_{B}\left(B^{H}\right)$.

By Theorem 3.5, we can obtain a relationship between the class of $H^{*}$-Galois Hirata extensions and the class of $H^{*}$-Galois Azumaya extensions which were studied in $[3,7]$.

COROLlary 3.6. Let $B$ be an $H^{*}$-Galois Azumaya extension of $B^{H}$. Then $B$ is an $H^{*}$-Galois Hirata extension of $B^{H}$ if and only if $C=C^{H}$.

Proof. $(\Rightarrow)$ Since $B$ is an $H^{*}$-Galois Hirata extension of $B^{H}, V_{B}\left(B^{H}\right)$ is an Azumaya algebra over $C$ and a left $H$-Galois extension of $C$ (see [8, Theorem 2.6]). Hence, $V_{B}\left(V_{B}\left(B^{H}\right)\right)=B^{H}$ (see [8, Lemma 2.5]). Thus, $C \subset B^{H}$; and so $C=$ $C^{H}$.

$(\Leftarrow)$ Since $B$ is an $H^{*}$-Galois Azumaya extension of $B^{H}, V_{B}\left(B^{H}\right)$ is separable over $C^{H}$ (see [3, Lemma 4.1]). Since $B$ is an $H^{*}$-Galois Azumaya extension of $B^{H}$ again, $V_{B}\left(B^{H}\right)$ is an $H^{*}$-Galois extension of $\left(V_{B}\left(B^{H}\right)\right)^{H}$ (see [3, Lemma 4.1]), so both $B^{H} \cdot V_{B}\left(B^{H}\right)$ and $B$ are $H^{*}$-Galois extensions of $B^{H}$ such that $B^{H} \cdot V_{B}\left(B^{H}\right) \subset$ $B$. Hence, $B^{H} \cdot V_{B}\left(B^{H}\right)=B$ by Lemma 3.1. This implies that the center of $V_{B}\left(B^{H}\right)$ is $C$. But by hypothesis, $C=C^{H}$, so $V_{B}\left(B^{H}\right)$ is an Azumaya $C$-algebra. Hence, $V_{B}\left(B^{H}\right)$ is a Hirata separable extension of $C$. But $B=B^{H} \cdot V_{B}\left(B^{H}\right) \cong B^{H} \otimes_{C}$ $V_{B}\left(B^{H}\right)$ as Azumaya $C$-algebras, so $B$ is a Hirata separable extension of $B^{H}$. Thus, $B$ is an $H^{*}$-Galois Hirata extension of $B^{H}$.

COROLlARY 3.7. Let $B$ be an $H^{*}$-Galois Hirata extension of $B^{H}$. Then $B$ is an $H^{*}$-Galois Azumaya extension of $B^{H}$ if and only if $B$ is an Azumaya $C^{H}$-algebra. 
Proof. $(\Rightarrow)$ Since $B$ is an $H^{*}$-Galois Azumaya extension of $B^{H}, B^{H}$ is an Azumaya $C^{H}$-algebra and $B$ is separable over $B^{H}$ (see [3, Theorem 3.4]). Hence, $B$ is separable over $C^{H}$ by the transitivity of separable extensions. But $B$ is an $H^{*}$-Galois Azumaya extension of $B^{H}$ and an $H^{*}$-Galois Hirata extension of $B^{H}$ by hypothesis, so $C=C^{H}$ by Corollary 3.6. This implies that $B$ is an Azumaya $C^{H}$-algebra.

$(\Leftarrow)$ By hypothesis, $B$ is an Azumaya $C^{H}$-algebra. Hence, $C=C^{H}$. But $B$ is an $H^{*}$-Galois Hirata extension of $B^{H}$, so $V_{B}\left(B^{H}\right)$ is an Azumaya subalgebra of $B$ over $C$ by Theorem 3.5(3). Since $B$ is an $H^{*}$-Galois Hirata extension of $B^{H}$ again, $B$ is a Hirata separable extension of $B^{H}$ and a finitely generated and projective module over $B^{H}$. Thus, $V_{B}\left(V_{B}\left(B^{H}\right)\right)=B^{H}$ (see [8, Lemma 2.5]); and so $B^{H}\left(=V_{B}\left(V_{B}\left(B^{H}\right)\right)\right)$ is an Azumaya subalgebra of $B$ over $C^{H}$ by the commutator theorem for Azumaya algebras (see [1, Theorem 4.3, page 57]). This proves that $B$ is an $H^{*}$-Galois Azumaya extension of $B^{H}$.

4. Invariant subalgebras. For an $H^{*}$-Galois Hirata extension $B$ as given in Theorem 3.5, let $A$ be a subalgebra of $B^{H}$ over $C$ such that $B^{H}$ is a projective Hirata separable extension of $A$ and contains $A$ as a direct summand as an $A$ bimodule. In this section, we show that $V_{B^{H}}(A)$ is the $H$-invariant subalgebra of a separable subalgebra $D$ in $B$ over $C$, that is, $D^{H}=V_{B^{H}}(A)$. We denote by $\mathscr{S}$ the set $\left\{A \mid A\right.$ is a subalgebra of $B^{H}$ over $C$ such that $B^{H}$ is a projective Hirata separable extension of $A$ and contains $A$ as a direct summand as an A-bimodule\}.

LEMмA 4.1. Let $B$ be an $H^{*}$-Galois Hirata extension of $B^{H}$. For any $A \in \mathscr{Y}$, $V_{B}(A)$ is an $H$-submodule algebra of $B$ and separable over $C$, and $\left(V_{B}(A)\right)^{H}=$ $V_{B^{H}}(A)$ which is a separable C-algebra.

Proof. Since $A \in \mathscr{Y}, B^{H}$ is a projective Hirata separable extension of $A$ and contains $A$ as a direct summand as an $A$-bimodule. But $B$ is an $H^{*}$-Galois Hirata extension of $B^{H}$, so $B$ is a projective Hirata separable extension of $B^{H}$. Hence, by the transitivity property of projective Hirata separable extensions, $B$ is a projective Hirata separable extension of $A$. Also $B^{H}$ is a direct summand of $B$ as a $B^{H}$-bimodule by Lemma 3.2, so $A$ is a direct summand of $B$ as an $A$-bimodule. Thus, $V_{B}(A)$ is a separable algebra over $C$ (see [6, Theorem 1]). Moreover, it is clear that $\left(V_{B}(A)\right)^{H}=V_{B^{H}}(A)$, so $V_{B^{H}}(A)$ is a separable $C$-algebra (see Corollary 3.4 and [6, Theorem 1]).

Next we want to show which separable subalgebra of $B^{H}$ over $C$ is an $H$ invariant subring of an $H$-submodule algebra in $B$. Let $\mathscr{T}=\{E \subset B \mid E$ is a separable $C$-subalgebra of $B^{H}$ and satisfies the double centralizer property in $B^{H}$ such that $\left.V_{B^{H}}(E) \in \mathscr{S}\right\}$. Next we show that for any $E \in \mathscr{T}, E$ is the $H$-invariant subring of an $H$-submodule algebra $D$ in $B$ which is separable over $C$.

THEOREM 4.2. Let $E$ be in $\mathscr{T}$. Then there exists an $H$-submodule algebra $D$ in $B$ which is separable over $C$ such that $D^{H}=E$. 
Proof. Since $E$ is in $\mathcal{T}, V_{B^{H}}(E)$ is in $\mathscr{S}$ such that $V_{B^{H}}\left(V_{B^{H}}(E)\right)=E$. Now by Lemma 4.1, $V_{B}\left(V_{B^{H}}(E)\right)$ is an $H$-submodule algebra of $B$ and separable over $C$ such that $\left(V_{B}\left(V_{B^{H}}(E)\right)\right)^{H}=V_{B^{H}}\left(V_{B^{H}}(E)\right)$. But $V_{B^{H}}\left(V_{B^{H}}(E)\right)=E$, so

$$
\left(V_{B}\left(V_{B^{H}}(E)\right)\right)^{H}=E \text {. }
$$

Let $D=V_{B}\left(V_{B^{H}}(E)\right)$. Then $D$ satisfies the theorem.

By Theorem 4.2, we obtain an expression for the separable $H$-submodule algebra $D$ for a given $E$ in $\mathscr{T}$.

COROLLARY 4.3. By keeping the notations as given in Theorem 4.2, let $Z$ be the center of $E$. Then $D \cong E \otimes_{Z} V_{D}(E)$ as Azumaya $Z$-algebras.

Proof. Since $E$ satisfies the double centralizer property in $B^{H}, V_{B^{H}}\left(V_{B^{H}}(E)\right)$ $=E$. Hence, the centers of $E$ and $V_{B^{H}}(E)$ are the same $Z$. Similarly as given in the proof of Lemma 4.1, since $V_{B^{H}}(E)$ is in $\mathscr{Y}, B\left(=B^{H} \cdot V_{B}\left(B^{H}\right)\right)$ is a projective Hirata separable extension of $V_{B^{H}}(E)$ and contains $V_{B^{H}}(E)$ as a direct summand as a $V_{B^{H}}(E)$-bimodule by the transitivity property of projective Hirata separable extensions and the direct summand conditions. Thus, $V_{B^{H}}(E)$ satisfies the double centralizer property in $B$, that is, $V_{B}\left(V_{B}\left(V_{B^{H}}(E)\right)\right)=V_{B^{H}}(E)$. This implies that the centers of $V_{B^{H}}(E)$ and $V_{B}\left(V_{B^{H}}(E)\right)$ are the same. Therefore, $D$ and $E$ have the same center $Z$. Noting that $D$ and $E$ are separable $C$-algebras by Theorem 4.2, we conclude that $E\left(=D^{H}\right)$ is an Azumaya subalgebra of $D$ over $Z$; and so $D \cong E \otimes_{Z} V_{D}(E)$ as Azumaya $Z$-algebras (see [1, Theorem 4.3, page 57$]$ ).

REMARK 4.4. When $B$ is an $H^{*}$-Galois Azumaya extension of $B^{H}$, the correspondence $A \rightarrow V_{B}(A)$ as given in Lemma 4.1 recovers the one-to-one correspondence between the set of separable subalgebras of $B^{H}$ and the set of $H^{*}$-Galois extensions in $B$ containing $V_{B}\left(B^{H}\right)$ as given in [3].

ACKNOWLEDGMENTS. This paper was written under the support of a Caterpillar Fellowship at Bradley University. The authors would like to thank Caterpillar Inc. for the support.

\section{REFERENCES}

[1] F. DeMeyer and E. Ingraham, Separable Algebras over Commutative Rings, Lecture Notes in Mathematics, vol. 181, Springer-Verlag, New York, 1971.

[2] H. F. Kreimer and M. Takeuchi, Hopf algebras and Galois extensions of an algebra, Indiana Univ. Math. J. 30 (1981), no. 5, 675-692.

[3] M. Ouyang, Azumaya extensions and Galois correspondence, Algebra Colloq. 7 (2000), no. 1, 43-57.

[4] K. Sugano, On centralizers in separable extensions, Osaka J. Math. 7 (1970), 29-40.

[5] _ Note on separability of endomorphism rings, J. Fac. Sci. Hokkaido Univ. Ser. I 21 (1970/1971), 196-208.

[6] _ On centralizers in separable extensions. II, Osaka J. Math. 8 (1971), 465469. 
[7] G. Szeto and L. Xue, On Hopf DeMeyer-Kanzaki Galois extensions, Int. J. Math. Math. Sci. 2003 (2003), no. 26, 1627-1632.

[8] K.-H. Ulbrich, Galoiserweiterungen von nicht-kommutativen ringen [Galois extensions of noncommutative rings], Comm. Algebra 10 (1982), no. 6, 655-672 (German).

George Szeto: Department of Mathematics, Bradley University, Peoria, IL 61625, USA E-mail address: szeto@hi11top.brad1ey.edu

Lianyong Xue: Department of Mathematics, Bradley University, Peoria, IL 61625, USA E-mail address: 1xue@hi11top.brad1ey.edu 


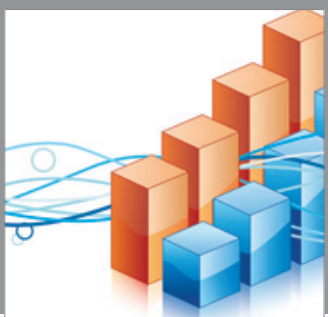

Advances in

Operations Research

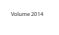

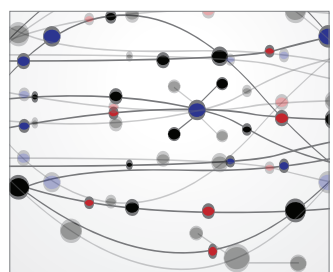

\section{The Scientific} World Journal
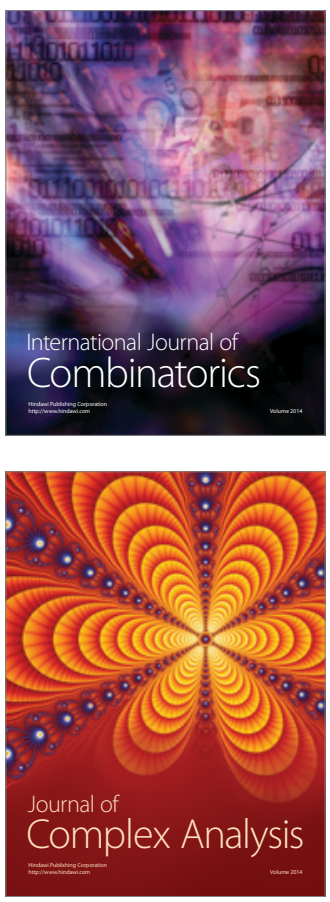

International Journal of

Mathematics and

Mathematical

Sciences
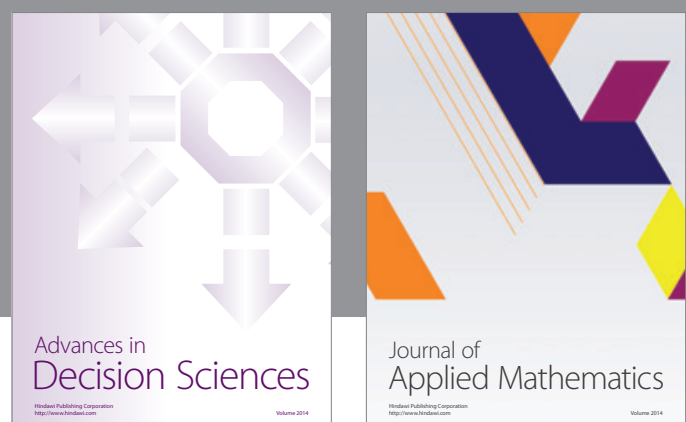

Journal of

Applied Mathematics
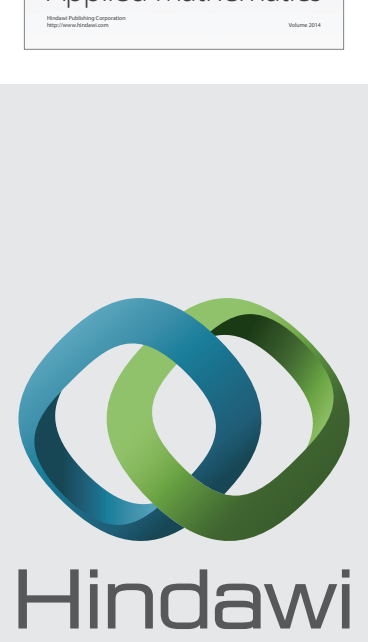

Submit your manuscripts at http://www.hindawi.com
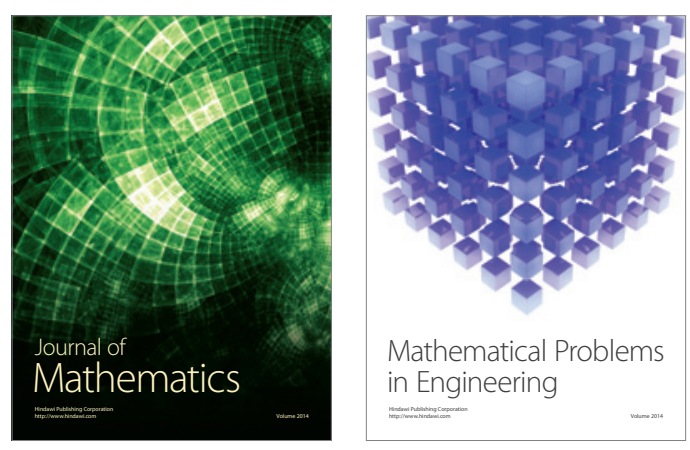

Mathematical Problems in Engineering
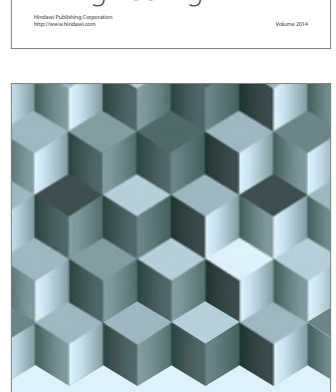

Journal of

Function Spaces
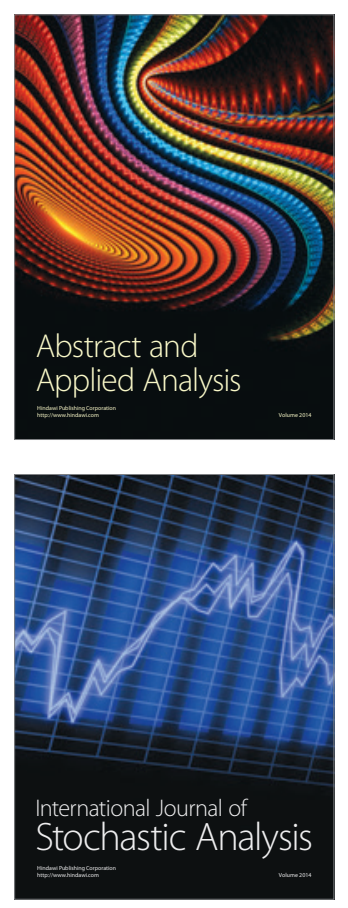

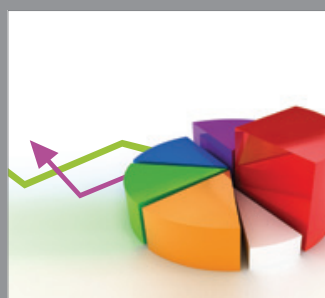

ournal of

Probability and Statistics

Promensencen
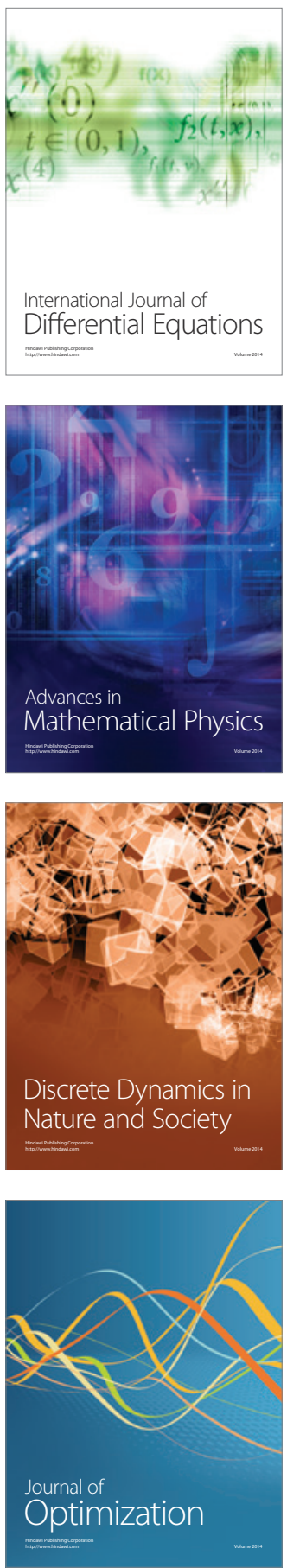